\title{
Toward A Better Understanding of Perceived Complexity in Music: A Commentary on Eerola (2016)
}

\author{
ELIZABETH HELLMUTH MARGULIS \\ University of Arkansas
}

\begin{abstract}
Eerola (2016) evaluates models of musical complexity based on expectancy violation and information theory. This commentary notes the deep relationship between these two phenomena, and argues for a more active partnership between computational and psychological approaches in understanding perceptions of musical complexity.
\end{abstract}

Submitted 2016 February 10; accepted 2016 March 5.

KEYWORDS: complexity, expectation, commentary

COMPLEXITY is a perceptually accessible aspect of musical experience. When I ask students without previous formal training to talk explicitly about what they heard in a piece, they start by talking about affect - how the music felt, and what it was trying to express. When prodded to consider structural aspects, they tend to speak most readily about the simplicity or complexity of various dimensions. Perceptions of complexity, it seems, are rather intuitive and reportable, even for people without formal training in music.

The datasets analyzed by Eerola in the target article take advantage of this reportability-they consist of explicit complexity ratings for a diverse set of melodies, ranging from isochronous tone rows to pop tunes to sub-Saharan African folk melodies. Eerola used computational models to understand whether expectancy-violation or information-theoretic approaches better explain the relationship between melodic structure and perceptions of complexity.

The distinction between the two types of model is not entirely clear. Three of the 8 parameters in the expectancy-violation model rely on entropy, a classic information theory measure. Eerola addresses this overlap in part by offering an alternative pared-down expectancy model consisting only of the top three most predictive parameters: tonal ambiguity, pitch proximity, and rhythmic variation. Yet these parameters seem to depend on statistical distributions as much as the information-theoretic ones: since perceived tonal hierarchies broadly match the statistical distributions of pitches in tonal music (Krumhansl, 1990), perceptions of tonal ambiguity (operationalized as the inverse of the mean stability of the tones in an excerpt) in fact could be argued to depend on statistical learning. Similarly, rhythmic variation is defined as the variance of note durations within a melody: a straightforwardly statistical measure. And finally, people who have worked on pitch proximity disagree about the extent to which it reflects a hard-wired tendency or an abstraction of the statistical properties of music to which listeners have been exposed (Huron, 2006; Margulis, 2007).

This confusion regarding the origin of the invoked parameters is not unique to Eerola's paper; on the contrary, it reflects a broader debate about the origins of human responses to music. What aspects are relatively invariant from listener to listener, and what aspects depend on enculturation, including statistical learning? Eerola is no stranger to this question, having published an influential paper on cross-cultural perceptions of musical complexity (Eerola et al., 2006). In the current paper, he notes that his models perform the poorest for two corpuses that likely "operate under different aesthetic premises": the one consisting of tone rows, and the one consisting of sub-Saharan African folk melodies. Since these stimuli were likely unfamiliar to participants in North America and Scandinavia, it's unclear what processes participants may have used to assess complexity. Encountering them for the first time in a laboratory setting, they didn't have a chance to absorb a set of aesthetic desiderata and norms in the way that normally happens implicitly with music exposure in social and real-life contexts. For example, participants may have started out trying to map norms derived from everyday listening to these corpuses, but gradually shifted across the course of the session to rating the stimuli in terms of the norms established by the melodies they'd heard in the experiment. Repertoire-specific intuitions likely influence ratings in broader ways as 
well. For example, if a certain style is ordinarily rhythmically complex, this feature-taken for grantedmay recede into the background, and complexity in other domains-like pitch and contour-might rise to the fore and disproportionately influence ratings; however if the repertoire were being encountered for the first time without any foreknowledge of this characteristic, perceptions of rhythmic complexity might dominate.

Questions about the role of enculturation in perceptions of complexity seem best answerable by a close partnership between behavioral research and computer modeling. Because the distinction between expectancy-violation and information-theoretic frameworks seems insufficiently stark, it might be more productive to investigate the malleability of complexity ratings with experience. For example, how might the ratings of complexity in sub-Saharan African folk melodies differ between a group of Western participants hearing them for the first time, a group of Western participants who had previously taken part in a series of exposure sessions familiarizing them with this repertoire, and a group of participants from this region? Rather than targeting the perennial question of whether judgments reflect innate principles or statistical learning, this sort of design could reveal the degree to which complexity ratings are shaped by norms abstracted over a lifetime of listening to all styles, or norms abstracted from familiarity—even shortterm familiarity-with the specific relevant corpus.

Furthermore, it might be possible to take real or constructed melodies and manipulate them according to the hypothesized factors - substituting pitches with different levels of tonal ambiguity, altering the rhythmic variation, introducing and collapsing skips so that pitch proximity is changed, or systematically varying the entropy along specific dimensions. Changes in the resultant complexity ratings could be analyzed in terms of changes in these underlying parameters.

Another avenue for future research might be the comparison of complexity perception across aesthetic domains. What parameters influence perceived complexity in visual art and fiction, and how do these relate to the parameters relevant in music? Designs investigating the role of expertise and enculturation could collect complexity ratings for various types of aesthetic stimuli from professional artists, musicians, and writers.

The rewards of better understanding perceived complexity could be significant. Although it is typically difficult to get people without formal training to talk about musical structure directly, rating perceived complexity doesn't seem to pose a problem. Experiments that cleverly manipulate aspects of musical structure, as well as aspects of musical familiarity and enculturation, can use complexity ratings to indirectly measure the way untrained listeners process musical structure. Moreover, since these percepts feed into aesthetic response and preference (cf. Rentfrow \& Gosling, 2003), the study of musical complexity can form an important foundation for the study of these more elusive phenomena.

\section{REFERENCES}

Eerola, T., Himberg, T., Toiviainen, P., \& Louhivuori, J. (2006). Perceived complexity of Western and African folk melodies by Western and African listeners. Psychology of Music, 34, 341-375. http://dx.doi.org/10.1177/0305735606064842

Eerola, T. (2016). Expectancy-violation and information-theoretic models of melodic complexity. Empirical Musicology Review, 11(1), 2-17. http://dx.doi.org/ 10.18061/emr.v11i1.4836

Huron, D. (2006). Sweet anticipation: Music and the psychology of expectation. Cambridge, MA: MIT Press.

Krumhansl, C. L. (1990). Cognitive foundations of musical pitch. New York, NY: Oxford University Press.

Margulis, E. H. (2007). Surprise and listening ahead: Analytic engagements with musical tendencies. Music Theory Spectrum, 29, 197-217. http://dx.doi.org/10.1525/mts.2007.29.2.197

Rentfrow, P. J., \& Gosling, S. D. (2003). The do re mi's of everyday life: The structure and personality correlates of music preferences. Journal of Personality and Social Psychology, 84, 1236-1256. http://dx.doi.org/10.1037/0022-3514.84.6.1236 